\title{
ESBOÇO DA MORFOLOGIA DA LÍNGUA SURUÍ- AIKEWÁRA, COM BASE NO CLÁSSICO TRABALHO DE RODRIGUES “A ESTRUTURA DO TUPINAMBÁ”
}

\section{A SKETCH OF THE SURUÍ-AIKEWÁRA MORPHOLOGY, BASED ON THE RODRIGUES' CLASSICAL WORK “A ESTRUTURA DO TUPINAMBÁ”}

\author{
Jorge Domingues Lopes \\ Universidade Federal do Pará, UFPA, Tocantins/Cametá, PA, Brasil \\ Laboratório de Línguas e Literaturas Indígenas da Universidade de Brasília
}

Resumo: Este artigo apresenta uma descrição de aspectos da morfologia da língua SuruíAikewára, língua pertencente ao subramo IV da família linguística Tupí-Guaraní (Rodrigues 1984/1985), tendo como referência o clássico estudo de Rodrigues (2010 [1981]), "A estrutura do Tupinambá".

Palavras-chave: Língua Suruí-Aikewára; Morfologia; Família Tupí-Guaraní.

Abstract: This article presents a description of morphological aspects of the Suruí-Aikewára language, which belongs to the subgroup IV of the Tupí-Guaraní family (Rodrigues 1984/1985). This article has as reference the classical work of Rodrigues (2010 [1981]), "A estrutura do Tupinambá”.

Keywords: Surui-Aikewara Language; Morphology; Tupi-Guarani family.

\section{Introduçáo}

Este trabalho apresenta uma análise de aspectos da morfologia da língua indígena Suruí-Aikewára, também conhecida como Mudjetíre ou Suruí do Tocantins, baseada no esboço detalhado da estrutura morfológica da língua Tupinambá ${ }^{1}$, por Rodrigues (2010 [1981], p. 11-42)².

\footnotetext{
${ }^{1}$ Segundo Rodrigues, esse trabalho de descrição da morfologia do Tupinambá foi iniciado na década de 1950 e concluído na década de 1980, com revisôes na década de 1990.

${ }^{2}$ Nesse trabalho, Rodrigues (2010, p. 11-12) trata da "divisão dos morfemas em afixos e raízes e [...] da classificação das raízes em função de sua combinação com os diferentes afixos", e também da reduplicaçẫo. Ele ainda classifica as raízes "segundo sua combinação ou não com os prefixos relacionais" e demonstra "as possibilidades do processo de composiçáo em Tupinambá", tratando, por fim, "das duas grandes classes de palavras identificáveis nessa língua - nomes e verbos".
} 
O Tupinambá e o Suruí, embora classificados em sub-ramos distintos da família linguística Tupí-Guaraní (RODRIGUES, 1984; 1985, p. 39-40; 45-46), são línguas gramaticalmente conservadoras, compartilhando uma alta percentagem de traços estruturais reconstruíveis para o Proto-TupíGuaraní.

Há ainda o fato de que o citado trabalho de Rodrigues tenha se consolidado como referência a inúmeros outros trabalhos descritivos de línguas Tupí-Guaranî́, os quais têm adotado sistematicamente a terminologia por ele proposta na descrição de modos verbais, de nominalizadores, de séries pronominais, de aspecto/modo de ação e de expressóes de modalidade, além dos processos de composição e de reduplicação típicos dessas línguas ${ }^{4}$.

\section{A morfologia da língua}

Os morfemas do Suruí-Aikewára classificam-se em afixos e raizes. Os afixos sáo prefixos flexionais - relacionais e pessoais - e derivacionais - nominalizadores, causativos e reflexivos. Os sufixos são, também, flexionais - causais, modais e de negação - e derivacionais - endocêntricos e exocêntricos.

A língua faz uso de reduplicação (monossilábica e dissilábica) e de composição (determinativa, atributiva e objetiva). As suas classes de temas dividem-se em três classes (Classe I, Classe II e Classe III), de acordo com a sua distribuição, relativa aos prefixos relacionais.

\subsection{Morfemas}

Apresento, nesta primeira parte, a descrição morfológica da língua Suruí e, em seguida, trato da caracterização das palavras (nomes, verbos e partículas) nessa língua.

\footnotetext{
${ }^{3}$ Ver, por exemplo, Jensen (1984, p. 75-117) e Cabral (2000, p. 233-262).

${ }^{4}$ Há também de se destacar a contribuição de Neves (1999), que descreveu aspectos da morfossintaxe da língua Suruí-Aikewára.
} 


\subsubsection{Afixos: Prefixos}

\subsubsection{Prefixos relacionais}

Segundo Cabral (2001) e Cabral Rodrigues e Franceschini (2013, p. 402), os prefixos relacionais constituem "um conjunto de prefixos que expressam a dependência sintática de um tema relativo com respeito ao seu determinante". Essa classe de prefixos pode fazer referência ao contexto gramatical ou ao contexto pragmático. Em Suruí, identificamos os seguintes prefixos relacionais, adaptados de Rodrigues (2010):

a) Prefixos relacionais que fazem referência ao contexto gramatical

$$
\begin{aligned}
& \left(u-\sim u s^{-}\right) \infty w-\quad \text { "O determinante de um nome [...] é } \\
& \text { se- } \quad \text { "O determinante de um verbo [...] é } \\
& \text { idêntico ao sujeito." } \\
& \text { so- } \quad \text { mente com o sujeito." } \\
& i-\infty \varnothing-\infty h \text { - } \quad \text { "O determinante é diferente do sujeito } \\
& \text { e distinto do falante e do ouvinte." } \\
& (r-\sim n-) \infty \varnothing- \\
& \text { "O determinante é a locução nominal } \\
& \text { contígua (imediatamente precedente)." }
\end{aligned}
$$

b) Prefixos relacionais que fazem referência ao contexto pragmático

$$
\begin{aligned}
& \text { uru- "O determinante é o ouvinte, sendo sujeito o } \\
& \text { falante ( } \pm \text { terceira pessoa)" } \\
& p \varepsilon-\quad \begin{array}{l}
\text { "O determinante é o ouvinte mais outrem, } \\
\text { sendo sujeito o falante ( } \pm \text { terceira pessoa)" }
\end{array} \\
& \text { "O determinante é ser humano indefinido": } \\
& t-\infty \varnothing-\infty \quad t-\varepsilon h a \text { 'olho de gente (ou de muitos)', } t \text {-aku } \\
& \text { ?- } \infty p \text { - > m- 'quentura de gente', } t \text {-uwi 'sangue de gente (ou } \\
& \text { de muitos)'; } m \text { ' 'mão de gente (ou de muitos)', } \\
& \varnothing \text {-pir?a 'fígado de gente (ou de muitos)' }
\end{aligned}
$$


1.1.1.2 Prefixos pessoais

\begin{tabular}{|c|c|c|}
\hline & $a-\infty w \varepsilon-$ & $\begin{array}{l}\text { a-wirgg 'eu roço', a-kupir 'eu capino'; } \\
\text {..we-ho-w 'indo eu' }\end{array}$ \\
\hline & $\varepsilon r \varepsilon-\infty \varepsilon-$ & $\begin{array}{l}\varepsilon r \varepsilon-t \dot{t r i t y g} \text { 'eu acordo', } \varepsilon r \varepsilon-k \varepsilon r \text { 'eu durmo'; } \\
\varepsilon-s \varepsilon-m u \text {-haku?i 'tenha cuidado' }\end{array}$ \\
\hline 13 & иги- & $\begin{array}{l}\text { uгu-asesmənəg 'nós (excl.) cortamos', } \\
\text { uгu-nups 'nós (incl.) batemos' }\end{array}$ \\
\hline 23 & $p \varepsilon-$ & $\begin{array}{l}\text { pe-suka 'eu mato', pe-wuhyj 'eu carrego', } \\
\text { pe-raha 'eu levo' }\end{array}$ \\
\hline 12(3) & $s a-$ & $\begin{array}{l}s a-k \varepsilon r \text { 'nós (incl.) dormimos', sa-ha 'nós } \\
\text { (incl.) vamos' }\end{array}$ \\
\hline & $u-\infty w-$ & u-pihig 'ele pega'; w-eraha 'ele leva' \\
\hline
\end{tabular}

Os dados apresentados anteriormente fundamentam o seguinte quadro, que leva em consideração a perspectiva de focalização do falante e do ouvinte.

\section{Quadro 1 - Matriz componencial dos prefixos pessoais em suruí, baseado na proposta de Rodrigues (2010, p. 13)}

\begin{tabular}{|c|c|c|c|c|}
\cline { 3 - 5 } \multicolumn{2}{c|}{} & \multicolumn{3}{c|}{ Oposição entre Falante e Ouvinte } \\
\cline { 2 - 5 } \multicolumn{2}{c|}{} & \multicolumn{2}{|c|}{+} & \multirow{2}{*}{-} \\
\cline { 3 - 5 } \multicolumn{2}{c|}{} & Falante & Ouvinte & \\
\hline \multirow{3}{*}{$3^{\text {3a }}$ pessoa focal } & - & $a-$ & $\varepsilon r \varepsilon-$ & $s a-$ \\
\cline { 2 - 5 } & + & $u r u-$ & $p \varepsilon-$ & $u-$ \\
\hline
\end{tabular}




\subsubsection{Prefixos derivacionais ${ }^{5}$}

a) Prefixos nominalizadores

A língua Suruí possui um único prefixo 'nominalizador de objeto' $\varepsilon m i-$.

1 ne remiriko ripo uapukaj ne upe

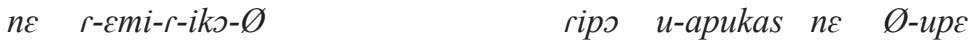

$2 R^{l}$-NMLZ-C.COM-estar.em.mov.-ARG DUB 3 -gritar $2 \quad R^{l}$-DAT

'a que faz você ficar com ela (sua esposa) está gritando para vocề

b) Prefixo causativo

O prefixo causativo simples do Suruí tem a forma $m u$-.

2 esemuhaku'i ke! ma'esawara rapo ne u'u

$\varepsilon$-se-mu-haku?i $\quad \mathrm{k} \varepsilon$ ma?csawar-a rapo $\mathrm{n} \varepsilon \quad \varnothing$-u?u

2-REFL-CAUS-cuidado DS cachorro-ARG POSS $2 \quad \mathrm{R}^{1}$-morder

'tenha cuidado! o cachorro vai te morder'

O prefixo causativo comitativo do Suruí tem as formas $r-\sim$ er- $\sim$ ero- $\sim$ ro-.

3 mowi pa'e ererur ma'ea ro'o?

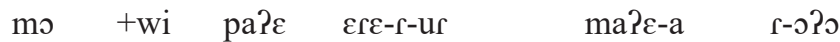

onde +ABL PERG.I 2-C.COM-trazer caça-ARG $\mathrm{R}^{1}$-carne

'de onde você trouxe esta carne de caça?'

\footnotetext{
${ }^{5}$ Abreviaturas: 1 = primeira pessoa; 2 = segunda pessoa do singular; 13 = primeira pessoa exclusiva; 23 = segunda pessoa do plural; 3 = terceira pessoa do singular; $\mathrm{ABL}=$ ablativo; $\mathrm{ARG}$ = argumento; $\mathrm{AT} . \mathrm{I}=$ atestado pelo locutor/recente; $\mathrm{C} . \mathrm{COM}=$ causativo-comitativo; C.PREP = causativo-prepositivo; CAUS $=$ causativo COMPL $=$ completivo $;$ CORR $=$ correferencial $;$ DAT = dativo; $\mathrm{DECL}=$ declarativo; $\mathrm{DES}=$ desiderativo; $\mathrm{DIR}=$ direcional; $\mathrm{DUB}=$ dubitativo $; \mathrm{ERG}=$ ergativo; EXCL = exclusivo; GER = gerúndio; $\mathrm{INCL}=$ inclusivo; IND.II $=$ indicativo $\mathrm{ii} ; \mathrm{INT}=$ intensivo (intensificador); $\mathrm{LD}=$ locativo difuso; $\mathrm{LP}=$ locativo pontual; $\mathrm{N}=$ nome; $\mathrm{NMLZ}=$ nominalizador; NMLZ.AG = nominalizador de agente; PERG.I = pergunta $\mathrm{i}$; PERMISS = permissivo; PROIB = proibitivo PROJ = projetivo $\mathrm{R}^{1}=$ relacional de contiguidade $; \mathrm{R}^{2}=$ relacional de nãocontiguidade; $\mathrm{R}^{3}=$ relacional genérico e humano; $\mathrm{R}^{4}=$ relacional que marca um determinante genérico; RED = reduplicação; REFL = reflexivo; SUBJ = subjuntivo; TRANSL = translativo; vi = verbo intransitivo; vt = verbo transitivo.
} 


\subsubsection{Afixos: Sufixos}

\subsubsection{Sufixos flexionais}

a) Os sufixos flexionais causais da língua Suruí são os seguintes:

- $-a \sim-\varnothing$ (marca o caso argumentativo $)^{6}$

$4 \quad$ ne apina

ne $\varnothing$-apin-a

$2 \mathrm{R}^{\mathrm{I}}$-cabeça-ARG

'tua cabeça'

5 pe nuwya

pe n-uwi-a

$23 \mathrm{R}^{1}$-sangue-ARG

'sangue de vocês'

6 sawara usaruetewa'e

sawar-a u-saru- $\varepsilon$ t $\varepsilon$-wa? $\varepsilon-\varnothing$

onça-ARG 3-ser.brabo-INT-NMLZ-ARG

'a onça é braba'

- -amu - -ramu -namu (marca o caso translativo)

7 aha puta ri'a isukaw tasahuamu

a-ha puta ri?a i-suka-w tasahu-amu

1-ir PROJ DECL $\mathrm{R}^{2}$-matar-GER porcão-TRANSL

'eu vou para matar porcão' (talvez eu mate um porcão)

8 ure purumu'etaramu

ure Ø-puru-mu?c-tar-amu

$13 \mathrm{R}^{1}$-gente-fazer.dizer-NMLZ.AG-TRANSL

'nós somos professores'

\footnotetext{
${ }^{6}$ De acordo com Cabral, Silva e Andrade (2013, p. 3), o caso argumentativo "caracteriza-se por marcar tanto nomes e verbos em função de argumento, quanto por englobar as principais funçóes gramaticais: sujeito de verbos transitivos (A) e intransitivos $(\mathrm{S})$, objeto direto $(\mathrm{O})$ e objeto de posposiçôes". Há, na língua Suruí, a ocorrência regular desse caso. Indico ainda, para uma discussão mais aprofundada do tema, o artigo de Rodrigues (1996, 57-66), no qual ele registra sua nova análise, desenvolvida em 1990, do morfema - $a$ que, em seus trabalhos anteriores, fora rotulado de caso nominal.
} 
9 monamu puta pa'e ituri wahemamapa?

mo-namu puta pare i-tur-i w-ahe-ma-map-a

quando-TRANSL PROJ PERG.I R ${ }^{2}$-vir-IND.II 3 CORR-chegar-COMPL-RED-GER 'quando todos vão chegar?'

- $-p e$ (marca o caso locativo pontual)

10 ita iwewu wewuj ti pope

ita- $\varnothing$ i-wewu-wewus ti $\varnothing$-po-pe

pedra-ARG 3-ser.leve-RED $\quad 1 \quad \mathrm{R}^{1}$-mão-LP

'a pedra está leve na minha mão'

11 aiko ra'e wehe re pa'e erehow ka'a pe eataw

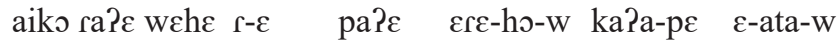

ontem $\mathrm{R}^{1}$-REL PERG.I 2-ir-GER mato-LP 2-andar-GER 'ontem você foi andar no mato'

- -imu (marca o caso locativo difuso)

12 'oga 'arimu

Pog-a Ø-Par-imu

casa-ARG $\quad \mathrm{R}^{1}$-superfície-LD

'em cima da casa'

b) Os sufixos flexionais modais da língua Suruí são os seguintes:

- $-a \sim-w \sim-t a$ (marca o modo gerúndio)

13 tapi'ira puta oho ka'a wi uhema

tapi?ir-a puta o-ho ka?a +wi u-hem-a

anta-ARG PROJ 3-ir mato ABL 3CORR-sair-GER

'a anta vai sair do mato'

14 pehe puta pesuka ma'ea pesehow?

peh $\varepsilon$ puta pe-suka ma?c-a pese-ho-w

23 PROJ 23-matar caça-ARG 23CORR-ir-GER

'vocês vão para matar aquelas caças?' 
15 aiko ra'e wehe rako aha ka'a pe weketa

aiko ra? $\varepsilon$ whe rako a-ha ka?a-pe we-ke(r)-ta

ontem AT.I 1-ir mato-LP 3CORR-dormir-GER

'ontem eu fui dormir no mato'

- $-i \sim-s$ (marca o modo indicativo II)

16 mowi pa'e ripo iture sawara?

$\begin{array}{lllll}\text { mo +wi pa?E } & \text { ripo } & \text { i-tur-i } & \text { sawar-a } \\ \text { onde ABL } & \text { PERG.I } & \text { DUB } & \mathrm{R}^{2} \text {-vir-IND.II } & \text { onça-ARG } \\ \text { 'de onde a onça vem?' } & & & \end{array}$

$17 \quad$ ka’awi puta ihoj tapi'ira

ka?a +wi puta i-ho-s tapi?is-a

mato +ABL PROJ $\mathrm{R}^{2}$-ir-IND.II anta-ARG

'do mato a anta vai sair'

- -amu - -ramu -namu (marca o modo subjuntivo)

18 Suta'ar sene 'arimu ihoramu puhi ke pe esaukar puhi

$\begin{array}{llll}\text { sutaPar-a } & \text { sene } & \varnothing \text {-Par-imu } & \text { i-ho-ramu } \\ \text { suta'ar-ARG } & 12(3) & \mathrm{R}^{1} \text {-superfície-LD } & \mathrm{R}^{2} \text {-ir-sUBJ }\end{array}$

puhi $\quad \mathrm{k} \varepsilon \quad \mathrm{p} \varepsilon \quad \varepsilon$-sa-ukar-a puhi

PROIB DES 23 2CORR-Ver-C.PREP-GER PROIB

'quando Suta’ar passar sobre nós, não amostrar (não apontar)'

- Não há marcas sufixais para o modo indicativo I na língua Suruí-Aikewára. Entretanto, a morfossintaxe do verbo - marcas pessoais, hierarquia referencial - nesse modo o distingue dos demais modos.

19 pehe rako ti nupo pe

pehe rako ti Ø-nupo pe

23 AT.I 1 R ${ }^{1}$-bater 2.ERG

'vocês bateram em mim' 
ure uru-apo ?-og-a

13 13-fazer $\mathrm{R}^{4}$-casa-ARG

nós fizemos estas casas

- Também náo existem marcas sufixais para o modo imperativo na língua Suruí. Esse modo recebe prefixos pessoais próprios, embora se combine com tais prefixos. Ressaltamos que a morfossintaxe do verbo no imperativo é também condicionada pela hierarquia referencial.

21 esuka

$\varepsilon$-suka

2-matar

'mata!'

22 pesahug peho

pe-sahug pe-ho

23-banhar 23-ir

'vão tomar banho!'

23 emono ma'esawara tukaru

$\begin{array}{llll}\text { pe-mon } \quad \text { ma?esawar-a } & \text { t(a) } & \text { u-karu } \\ \text { 23-dar cachorro-ARG } & \text { PERMIss } & \text { 3-comer } \\ \text { 'deem comida pro cachorro!' } & & \end{array}$

\subsubsection{Sufixos derivacionais}

a) endocêntricos

A língua Suruí-Aikewára também possui um conjunto de sufixos derivacionais que formam temas que mantêm a mesma classe da base, são eles:

- $-h u--u h u$ (intensivo)

tatu $+-h u \rightarrow$ tatuhu 'tatu grande'

tarciri $+-u h u \rightarrow$ tarciriuhu 'traíra grande'

ame? $+-u b u \rightarrow$ ame?auhu 'testículo grande' 
- $-2 i$ (atenuativo)

wira $+-P i \rightarrow$ wira?i 'pássaro pequeno'

akara $+-P i \rightarrow$ akara?i 'acará pequeno'

- - 2 itm (privativo)

tahi $+-\varepsilon$ Pim $\rightarrow$ tahic?im 'sem dor de gente'

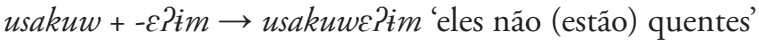

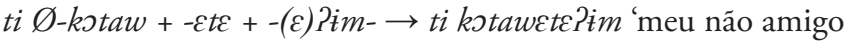

(meu inimigo)'

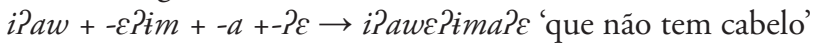

- -tus (coletivizador)

awaitahu + -tus $\rightarrow$ awaitahutus 'jovens'

awa?imon + -tus $\rightarrow$ awa?imontus 'velhos'

b) exocêntricos

Há vários sufixos derivacionais exocêntricos na língua Suruí que formam temas com classe diferente da classe da base, são eles:

- Nominalizadores de temas verbais

- -ar 'nome de agente'

$i-+$ putat $+-a r+-a \rightarrow$ iputatara 'aquele que quer, queredor'

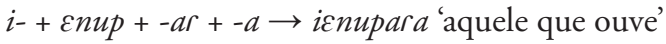

$i-+$ hest $+-a r+-a \rightarrow$ ihestara 'lavador'

- -aw -haw 'nome de circunstância'

$n \varepsilon \varnothing-k \varepsilon(r)+h a w+a \rightarrow n \varepsilon$ kchawa 'lugar de deitar de você'

putik $+a w \rightarrow$ putikaw 'lugar para cagar'

tata $+p \varepsilon k w a+(a) w \rightarrow$ tatap $\varepsilon k w a w$ 'instrumento para abanar o fogo'

so kitik $+a w+a \rightarrow s o$ kittkawa 'ralador de castanha' ('paxiúba') ${ }^{7}$

$\rightarrow$ 'liquidificador' (neologismo pós-contato)

$? \dot{i}+? u+a w+a \rightarrow ? \dot{t}$ ? $u a w a$ 'ingeridor de água' $\rightarrow$ 'copo'

(neologismo pós-contato)

\footnotetext{
${ }^{7}$ A paxiúba era usada como ralador de castanha-do-pará pelos Suruí. Com a chegada do liquidificador, a função de ralar a castanha ficou a cargo desse novo aparelho, que recebeu, por sua vez, a mesma denominação, so kytykawa, do antigo instrumento de ralar.
} 
- -pir 'nome de paciente'

$$
\begin{aligned}
& \text { suka }+ \text { pir } \rightarrow \text { sukapir 'o morto' } \\
& \text { nups }+ \text { pir } \rightarrow \text { nupspir 'o que foi batido' }
\end{aligned}
$$

- Nominalizadores de frases

- -ware 'nominalizador de predicado'

24 u'yahawa'e urusuka misara

u-Piaha-wa?e uru-suka misar-a

3-atravessar.o.rio-NMLZ.PRED 13-matar veado-ARG

'nós matamos o veado que atravessou o rio'

- -war 'nome de procedência'

Este nominalizador forma nomes de procedência construídos a partir de sintagmas posposicionais.

25 ti roga pupewara ripo mongaty oho

ti r-og-a Ø-pupe-war-a ripo mo Ø-kati o-ho

$1 \quad \mathrm{R}^{1}$-casa-ARG $\mathrm{R}^{1}$-dentro-NMLZ-ARG DUB onde $\mathrm{R}^{1}$-DIR 3-ir

'os que são de dentro de minha casa possivelmente foram na direção de algum lugar' (na minha casa não tem ninguém)

26 ka’apewara

ka?a-pe-war-a

mato-LP-NMLZ-ARG

'no mato ele foi'

- -wa?E 'nominalizador de circunstâncias'

27 Miho use'engar kwahawa'e

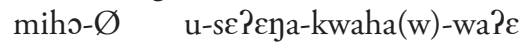
miho-ARG 3-cantador-conhecer-NMLZ

'Miho é um bom cantador' 
28 ti rirua iaturuewa'e

$$
\begin{aligned}
& \text { ti r-iru-a i-aturue-wa?c } \\
& 1 \quad \mathrm{R}^{1} \text {-camisa-ARG } \mathrm{R}^{2} \text {-curta-NMLZ } \\
& \text { 'minha camisa é a que está curta' }
\end{aligned}
$$

\subsubsection{Reduplicação}

Há dois tipos de reduplicação na língua Suruí-Aikewára, a monossilábica e a dissilábica, ambas indicando intensificaçáo da qualidade ou da ação/processo.

a) Reduplicação monossilábica

kons 'torto' $\rightarrow$ kononos 'muito torto (manco)'

akus 'eu caio' $\rightarrow$ akukus 'eu caio várias vezes'

b) Reduplicação dissilábica

amomon 'eu enrolo' $\rightarrow$ amomomomon 'eu enrolo várias vezes'

tapisar 'muitos (mais de quatro)' $\rightarrow$ tapisapisar 'muitos (em grande quantidade)'

apukas 'eu grito' $\rightarrow$ apukapukas 'eu grito ainda mais forte (ecoar)' amupen 'eu quebro' $\rightarrow$ amupemupen 'eu quebro em vários pedaços' aruPag 'eu viro' $\rightarrow$ aruParuPag 'eu viro várias vezes (girar)'

\subsection{Raízes}

Além dos afixos, a outra categoria analisada é a das raízes na língua Suruí.

\subsubsection{Classificação das raízes}

A classificação de raízes proposta por Rodrigues (2010, p. 16-17) leva em consideração tanto a possibilidade quanto a não possibilidade de combinação da raiz com afixos flexionais. Dessa forma, é possível identificar três classes de raízes:

- Classe I: combinável com o prefixo $i$ - do relacional $\mathrm{R}^{2}$.

- Subclasse Ia: raízes que não começam por /p/: combinam-se com o alomorfe $\varnothing$ - do $\mathrm{R}^{4}$. 
- Subclasse Ib: raízes que começam por /p/: combinam-se com o alomorfe $m$ - do $\mathrm{R}^{4}$.

- Classe II: combinável com os alomorfes $t$-, h- e $\varnothing$ - do relacional $\mathrm{R}^{2}$ (admite somente raízes iniciadas por vogal).

- Subclasse IIa: raízes que se combinam com o $\varnothing$ - do relacional $\mathrm{R}^{2} \mathrm{e}$ $t$ - do relacional $\mathrm{R}^{4}$.

- Subclasse IIb: raízes que se combinam com o $t$ - do relacional $\mathrm{R}^{2} \mathrm{e}$ $t$ - do relacional $\mathrm{R}^{4}$.

- Subclasse IIc: raízes que se combinam com o $t$ - / Ø- / h-do relacional $\mathrm{R}^{2}$ e $\varnothing-/ t$ - / h-do relacional $\mathrm{R}^{4}$.

- Subclasse IId: raízes que se combinam com o $\varnothing$ - do relacional $\mathrm{R}^{2}$ e $t-1$ $u$ - $\rightarrow \varnothing$ - do relacional $\mathrm{R}^{4}$.

- Classe III: náo combinável com prefixos relacionais (admite somente raízes nominais)

\section{Quadro 2 - Distribuiçáo dos prefixos das categorias $R^{1}$ a $R^{4}$, segundo as classes e subclasses das raízes}

\begin{tabular}{|c|c|c|c|c|c|c|}
\hline Classes & Subclasses & $\mathrm{R}^{1}$ & $\mathrm{R}^{2}$ & $\mathrm{R}^{3}$ & $\mathrm{R}^{4}$ & Exemplos \\
\hline \multirow[t]{2}{*}{ I } & a & $\varnothing-$ & $i-$ & $u-/ w-$ & $\varnothing-$ & $\begin{array}{c}\text { apin 'cabeça', 'aw } \\
\text { 'cabelo', } k J \text { 'roça', ker } \\
\text { 'dormir', pit?a 'fígado', } \\
\text { siw?a 'braço' }\end{array}$ \\
\hline & $\mathrm{b}$ & $\varnothing-$ & $i-$ & $u-$ & $p->m-$ & $\begin{array}{l}\text { po 'mão', pir 'pele', } \\
\text { purahas 'dançar', } \\
\text { pepuwir 'sovaco' }\end{array}$ \\
\hline \multirow{6}{*}{ II } & a.i & $r-/ n-$ & $\varnothing-$ & $u-/ u s-$ & $t-$ & $\begin{array}{l}\text { aku 'quente', cha 'olho', } \\
\text { cms 'pênis', } u w \dot{t} \text { 'sangue' }\end{array}$ \\
\hline & a.ii & $r-/ n-$ & $h-$ & $u-/$ us- & $t-$ & os 'dente' \\
\hline & a.ii & $r-/ n-$ & $b-$ & $u-/$ us- & $\varnothing-$ & sw 'folha' \\
\hline & b & $r-/ n-$ & $t-$ & $w-$ & $t-$ & $\begin{array}{l}u w \text { 'pai', a a'tr 'filho } \\
\text { (homem falando)' }\end{array}$ \\
\hline & c & $r-/ n-$ & $b-$ & $w$ & ?- & Pog 'casa' $u$ Pitw 'flecha' \\
\hline & d & $r-/ n-$ & $\varnothing$ & $w-$ & $t-\rightarrow \varnothing$ & $\begin{array}{l}\text { ckus 'cuia', puramor } \\
\text { 'peidar', puti 'cagar' }\end{array}$ \\
\hline
\end{tabular}




\begin{tabular}{|c|c|c|c|c|c|c|}
\hline III & - & - & - & - & - & $\begin{array}{c}\text { amənisu 'algodão', arar } \\
\text { 'arara', sawar 'onça', } \\
\text { kwarahy 'sol', wasn๖m } \\
\text { 'beija-flor' }\end{array}$ \\
\hline
\end{tabular}

Observaçáo: No caso do relacional que marca um determinante genérico $\left(\mathrm{R}^{4}\right)$ do tema $1 \mathrm{~b}$, existe, na língua Suruí, uma forma supletiva iniciada $\operatorname{com} n$.

\subsubsection{Composiçáo}

\subsubsection{Composição determinativa}

Neste tipo de composição há duas raízes nominais em que a primeira determina a segunda:

aru nIII 'sapo (esp.)' + po nIb 'mão' $\rightarrow$ arupo 'lit. mão do sapo $\rightarrow$ rastelo, garfo'

inata nIII 'coco' + rí nIII 'água' $\rightarrow$ inatari 'água de coco'

$k s m$ nIa 'seio' + íru nIII 'recipiente' $\rightarrow$ ksmiru nIa 'recipiente do

seio $\rightarrow$ sutiâ'

sakare nIII 'jacaré' + sitw nIa 'mandíbula' $\rightarrow$ sakareasitw nIII

'mandíbula de jacaré $\rightarrow$ cangalha'

\subsubsection{Composição atributiva}

(a) há duas raízes nominais, sendo que a primeira é determinada pela segunda.

ipira nIII 'peixe' + os nIIb 'dente' $\rightarrow$ ipiros nIII 'peixe dentudo $\rightarrow$ piranha'

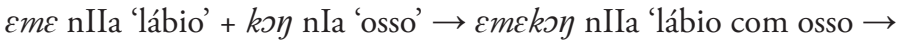
gengiva'

(b) há duas raízes, uma nominal e outra nominal descritiva; aqui a segunda determina a primeira.

misara nIII 'veado' + piron nIa 'vermelho' $\rightarrow$ misarapiroy nIII 'veado-vermelho'

sawara nIII 'onça' + pinima nIa 'pintado' $\rightarrow$ sawapinima nIII 'onça-pintada' 
(c) há duas raízes, uma nominal e outra verbal intransitiva, em que a segunda determina a primeira.

ipira n.III 'peixe' + ku? om v.intr 'levantar' + -aw nmlz $\rightarrow$ ipiraku? omawa n.III 'peixe levantado'

ipira n.III 'peixe' + wewe v.intr 'voar' + -wa? $\mathrm{nmlz} \rightarrow$ ipirau-

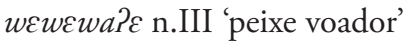

(d) há duas raízes, uma verbal (transitiva ou intransitiva) e outra nominal descritiva, em que a segunda determina a primeira.

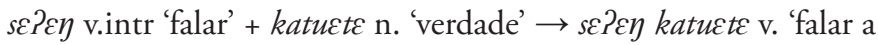
verdade'

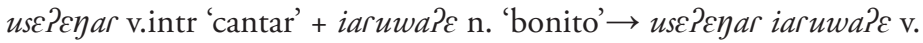
'o que canta bonito'

\subsubsection{Composição objetiva}

(a) há duas raízes, uma nominal e outra verbal transitiva, em que a primeira determina a segunda.

puru nIII 'gente' + suka vt 'matar' $\rightarrow$ purusuka 'matar gente' puru nIII 'gente' + mupise vt 'fazer bem' $\rightarrow$ purumupise 'fazer bem (pra) gente'

nami nIIa 'orelha' + kutuk vt 'furar' $\rightarrow$ namikutuk 'furar orelhar' ti nIIa 'nariz' + nupen vt 'quebrar' $\rightarrow$ timupen 'quebrar nariz'

\subsubsection{Composição mista}

Assim como no Tupinambá, na língua Suruí toda composição pode ser componente de uma nova composição.

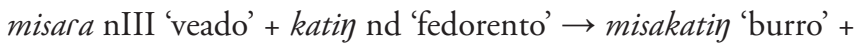
-rona sml 'parecido' $\rightarrow$ misakatinrona nIII 'parecido com burro fedorento $\rightarrow$ vaca, boi'

\subsection{Nomes e verbos}

Partindo da noção de classes de palavras da gramática tradicional, Payne (2006) afirma que, em muitas línguas, as duas principais classes são as dos nomes e dos verbos. Segundo esse autor, 
Para nomes e verbos, protótipos podem ser identificados em termos de significado. A classe dos NOMEs em qualquer língua inclui palavras que se referem a entidades DELIMITADAS ou INDIVIDUALIZADAS, por exemplo, 'árvore', 'montanha' 'mausoléu' etc. Estes são conceitos que não tendem a mudar muito ao longo do tempo, e que podem ser referidos repetidamente no discurso como a mesma coisa (PAYNE, 2006, p. 94).

Ambas as categorias, a do nome e a do verbo, existem na língua Suruí e, conforme observado por Payne (2006), nessa língua elas correspondem às formas mais recorrentes. A seguir, trato de cada uma delas, separadamente.

\subsubsection{Nomes}

Os nomes em Suruí são flexionáveis e, com exceção dos nomes relacionais, se combinam com sufixos casuais. Além disso, eles não se combinam com os prefixos pessoais, nem com os sufixos modais.

Rodrigues (2010) identifica, em Tupinambá, subclasses dos nomes: substantivos, nomes descritivos, paradigmas do indicativo I de nomes descritivos como núcleos de predicados e demonstrativos. Essas mesmas subclasses também estão presentes na língua Suruí.

\subsubsection{Substantivos}

De acordo com Rodrigues (2010, p. 21), esta subclasse dos substantivos é aberta, não possui restriçôes quanto à sua distribuição, ou seja, ela pode ocorrer como "determinante ou determinado nas locuçóes, como sujeito ou objeto nas oraçôes", como pode ser observado nos exemplos da língua Suruí, a seguir:

-Paradigmas deflexão casual: 1) -uwnIIb 'pai',2) -irunIa 'companheiro', 3) ka?a nIII 'mato', 4) kwar nIa 'buraco', 5) iptitun nIII 'noite', 6) Piwir nIa 'cintura'.

\section{Tabela 1 - Paradigmas de flexão casual}

\begin{tabular}{|c|c|c|c|c|c|c|}
\hline Caso & & & & & & \\
\hline Argumentativo & $-u w-a$ & iru-Ø & $k a ? a-\varnothing$ & $k w a r-a$ & ipitun-a & Piwir-a \\
\hline Translativo & $-u w-a m u$ & iru-namu & ka?a-ramu & kwar-amu & & Piwir-amu \\
\hline Loc. pontual & & & $k a a^{2} a-p \varepsilon$ & & & \\
\hline Loc. difuso & & & & & ipitun-amu & Piwir-imu \\
\hline
\end{tabular}


- Paradigmas de flexão determinativa com prefixos relacionais: 1) apin nIa 'cabeça', 2) ti nIa 'nariz', 3) po nIb 'mão', 4) $\varepsilon$ ha nIIa 'olho', 5) uw nIIb 'pai', 6) upiw nIIc 'flecha', 7) pe nIId 'caminho', ekus nIId 'cuia'.

Tabela 2 - Paradigmas de flexáo determinativa com prefixos relacionais

\begin{tabular}{|c|c|c|c|c|c|c|c|c|}
\hline Prefixos & & & & & & & & \\
\hline $\mathrm{D}=\mathrm{S}$ & u-apina & $u-t i$ & ว-ps & $w$-cha & $u s-u w a$ & $u$-upiwa & $w-a p \varepsilon$ & $w$-ckus \\
\hline$D \neq S$ & $i$-apina & $i-t i$ & $i-p s$ & $h-\varepsilon h a$ & $t-u w a$ & $t-u p \dot{t} w a$ & $h-a p \varepsilon$ & $h-\varepsilon k u s$ \\
\hline $\mathrm{D}=\mathrm{C}$ & $\varnothing$-apina & $\varnothing-t i$ & $\varnothing-p s$ & $r-\varepsilon h a$ & $r-u w a$ & r-upiwa & $r$-ape & r-ckus \\
\hline $\mathrm{D}=\mathrm{H}$ & $\varnothing$-apina & $\varnothing-t i$ & $m s$ & $t-\varepsilon h a$ & $t-u w a$ & ?-u?iwa & $t$-ape & $t-\varepsilon k u s \sim k u s$ \\
\hline
\end{tabular}

- Em Suruí, há três sufixos que expressam o estado de existência dos referentes de nomes, $-\varnothing$ 'atual', -rom 'prospectivo' $e-k w \varepsilon r$ 'retrospectivo'.

Tabela 3 - Formas - $r \supset m$ e $-K w \varepsilon r$

\begin{tabular}{|c|c|c|}
\hline ATUAL & RETROSPECTIVO & Prospectivo \\
\hline $\begin{array}{l}\varnothing-k \supset \text { nIa } \\
\text { 'roça' }\end{array}$ & $\begin{array}{l}\text { kəkwera } \\
\text { 'ex-roça' }\end{array}$ & $\begin{array}{c}\text { kərəm } \\
\text { 'que vai ser roça' }\end{array}$ \\
\hline $\begin{array}{l}\text { Ø-piwwi nIII } \\
\text { 'árvore' }\end{array}$ & $\begin{array}{l}\text { Piwipukwera } \\
\text { 'tronco' }\end{array}$ & $\begin{array}{c}\text { Piwipukurom } \\
\text { 'que vai ser tronco' }\end{array}$ \\
\hline $\begin{array}{c}\varnothing \text {-crckatara nIa } \\
\text { 'marido' }\end{array}$ & $\begin{array}{l}\text { crekatarera } \\
\text { 'viúva' }\end{array}$ & $\begin{array}{l}\text { crckatararəm } \\
\text { 'futura viúva' }\end{array}$ \\
\hline $\begin{array}{l}\text { Ø-sc?cy } \\
\text { 'fala' }\end{array}$ & $\begin{array}{l}\text { se?cyawera } \\
\text { 'recado' }\end{array}$ & $\begin{array}{c}\text { sc?cyarom } \\
\text { 'que vai ser recado' }\end{array}$ \\
\hline $\begin{array}{c}\text { Ø-cmircko nIa } \\
\text { 'esposa' }\end{array}$ & $\begin{array}{l}\text { cmirckokwera } \\
\text { 'ex-esposa' }\end{array}$ & $\begin{array}{l}\text { cmirckorom } \\
\text { 'futura esposa' }\end{array}$ \\
\hline
\end{tabular}

- Vocativos: Rodrigues (2010) identificou dois tipos de vocativo: vocativos substantivos e vocativos independentes.

Tabela 4 - Vocativos na língua suruí-aikewára

\begin{tabular}{|c|l|}
\hline \multirow{3}{*}{$\begin{array}{c}\text { Vocativos } \\
\text { substantivos }\end{array}$} & timiksy nIa 'pelve': timikoy! 'Pelve!' \\
\cline { 2 - 2 } & ikatu nIII 'macaxeira, o que é bom': ikatu! 'Macaxeira!' \\
\cline { 2 - 2 } & iwikatu nIII 'terra boa': iwikatu! 'Terra boa!' \\
\cline { 2 - 2 } & wasnom nIII 'beija-flor': wasnom! 'Beija-Flor!' \\
\hline
\end{tabular}




\begin{tabular}{|c|l|}
\hline \multirow{3}{*}{$\begin{array}{c}\text { Vocativos } \\
\text { independentes }\end{array}$} & mitum voc 'pai!', 'papai!' (mulher falando) \\
\cline { 2 - 2 } & na voc 'pai!', 'papai!' (homem falando) \\
\cline { 2 - 2 } & mibi voc 'mãe!', 'mamãe!' (mulher falando) \\
\cline { 2 - 2 } & inc voc 'mãe!', 'mamãe!' (homem falando) \\
\hline
\end{tabular}

\subsubsection{Nomes descritivos}

"Subclasse aberta, com restrições de distribuição; pode ocorrer como determinado nas locuçóes, como sujeito ou objeto nas oraçóes verbais e como núcleo de predicados descritivos" (RODRIGUES, 2010, p. 24): uriw 'alegre, alegria', uriwa 'alegria', uriwramu 'na qualidade de estar alegre', indicativo II uriw 'está alegre'.

- Paradigmas do indicativo I de nomes descritivos como núcleos de predicados: esses paradigmas incluem formas com os prefixos relacionais $i-\infty$

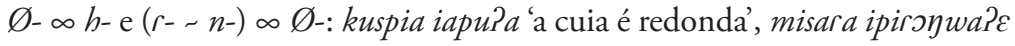
'o veado é vermelho'.

- Paradigmas do gerúndio de nomes descritivos como núcleos de predicados:

29 wakuwamu

w-akuw-amu

3-quente-GER

'ficando quente'

30 wurywamu

w-uriw-amu

3-alegre-GER

'ficando alegre'

\subsubsection{Numerais}

A língua Suruí possui palavras para expressar números até quatro, utilizando, para além disso, uma forma genérica, que pode ser reduplicada.

- usepese 'um'

- namukus 'dois'

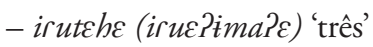

- irutchehik 'quatro' 
- tapisar 'muitos'

- tapisapisar 'muitos (indefinido)'

\subsubsection{Demonstrativos}

Os demonstrativos na língua Suruí constituem uma "subclasse fechada, com certas particularidades de distribuição morfológica e sintática" (RODRIGUES, 2010, p. 26), tomando como referência o próprio falante (próximo/afastado).

\section{Quadro 3 - Matriz componencial dos demonstrativos na língua suruí}

\begin{tabular}{|c|c|c|c|c|}
\hline & \multirow[b]{2}{*}{$\begin{array}{l}\text { Próximo do } \\
\text { falante }\end{array}$} & \multicolumn{2}{|c|}{ Afastado do falante } \\
\hline & & & $\begin{array}{l}\text { Próximo do } \\
\text { ouvinte }\end{array}$ & $\begin{array}{l}\text { Afastado do } \\
\text { ouvinte }\end{array}$ \\
\hline \multirow{2}{*}{ Determinado } & Visível & $\begin{array}{l}k s \text { (este, aqui) } \\
i k s \text { (daqui) } \\
\text { Paw (este aqui, } \\
\text { bem próximo } \\
\text { ao falante) } \\
\end{array}$ & & $\begin{array}{l}\text { kwes (aquele lá, } \\
\text { lugar) } \\
p \varepsilon \text { (esse lá) } \\
p \varepsilon w \text { (lá) }\end{array}$ \\
\hline & Invisível & aiks (este) & $\begin{array}{l}a ? \varepsilon \text { (esse, } \\
\text { aquele de } \\
\text { quem se fala) }\end{array}$ & $\begin{array}{l}\text { aikwes (aquele } \\
\text { lá) } \\
\text { aipe (esse lá) } \\
\text { aipew (lá) }\end{array}$ \\
\hline \multicolumn{2}{|c|}{ Indeterminado } & \multicolumn{3}{|c|}{ aimi (aquele) } \\
\hline
\end{tabular}

\subsubsection{Pronome}

Com relação aos pronomes, que constituem uma "subclasse fechada, com limitaçôes de distribuiçẫo morfológica e sintática" (RODRIGUES, 2010, p. 29) na língua Suruí, eles podem ser divididos em três séries:

- Série I

"Só ocorre como enunciado independente, como sujeito de oraçóes equativas e como sujeito enfático de outras oraçóes." 
- Série II "Ocorre como determinante em sintagmas nominais."

- Série III "Só ocorre como sujeito de oraçôes transitivas cujo objeto é ou inclui o falante." (RODRIGUES, 2010, p. 29)

\section{Quadro 4 - Matriz componencial dos pronomes (Série I)}

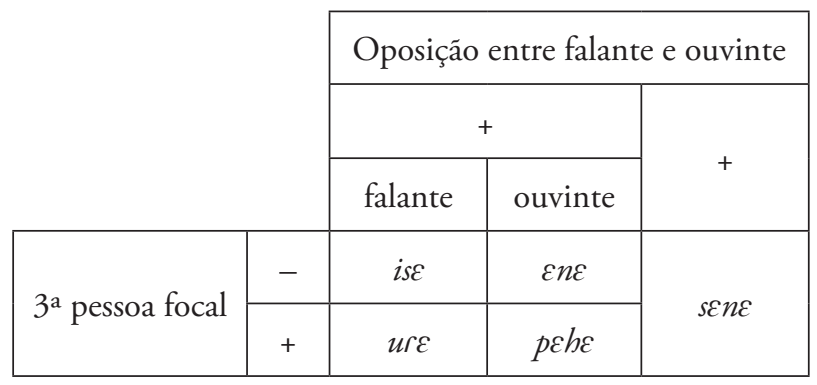

\section{Quadro 5 - As três séries de pronomes e o dativo}

\begin{tabular}{|c|c|c|c|c|}
\hline & & I & II & III \\
\hline falante $\pm 3^{\text {a }}$ pess. náo focal & 'eu’' & is $\varepsilon(\mathrm{s} \varepsilon)$ & ti & - \\
\hline falante $\pm 3^{\mathrm{a}}$ pess. focal & 'nós (excl.)' & ure & ґع & - \\
\hline ouvinte $\pm 3^{\mathrm{a}}$ pess. não focal & 'você' & $\varepsilon n \varepsilon(\mathrm{n} \varepsilon)$ & $\mathrm{n} \varepsilon$ & sepe \\
\hline ouvinte $\pm 3^{\mathrm{a}}$ pess. focal & 'vocês' & pehe & $\mathrm{p} \varepsilon$ & penche \\
\hline falante \pm ouvinte $\pm 3^{\text {a }}$ pess. focal & 'nós (incl.)' & scne & senc & - \\
\hline
\end{tabular}

\subsubsection{Relacional (posposiçôes)}

De acordo com Rodrigues (2010, p. 30), os relacionais (posposiçóes) constituem uma "subclasse fechada, com limitaçôes de distribuição morfológica e sintática", que "só se flexionam com os prefixos relacionais e só ocorrem formando complementos nas oraçóes". Essas formas ocorrem na língua Suruí, como pode ser observado nos exemplos a seguir:

$-p \varepsilon$, upe RIa 'dativo' 
- pире RIa 'dentro'

- upi RIb 'ao longo de, pelo'

- kati RIa 'na direção de'

- Enune RIIa 'na frente de'

\subsubsection{Verbo}

A classe dos verbos, de acordo com Rodrigues (2010, p. 31), forma uma "classe de palavras flexionadas que se combinam com os prefixos pessoais [...] e com os sufixos modais [...]”. E, tal como no Tupinambá, a língua Suruí distingue, também, duas subclasses de verbos, a dos intransitivos e a dos transitivos, como pode ser verificado nos dados abaixo.

\subsubsection{Verbos intransitivos}

A subclasse dos verbos intransitivos na língua Suruí "se combina com os prefixos pessoais unidos diretamente ao tema" (RODRIGUES, 2010, p. 31): ho vi Ia 'ir', aha vi Ia 'eu vou'; son vila 'correr', ason 'eu corro/corri'.

Por outro lado, quando esses verbos são combinados com "o sufixo modal de gerúndio [...], os verbos intransitivos ocorrem com o alomorfe" $w \varepsilon$ - e $\varepsilon$-dos prefixos $a$ - e $\varepsilon r \varepsilon-$ - ho vi Ia 'ir', wehow 'indo eu', $\varepsilon h o w$ 'indo você'.

\subsubsection{Verbos transitivos}

Os verbos transitivos da língua Suruí-Aikewára constituem uma "subclasse aberta, que se combina [...] com os prefixos pessoais e com os prefixos relacionais": esag vtIIa 'ver', acsag akuma? 'eu vejo o homem'; nupo vtla 'bater', ise raks runups 'eu bati em vocês dois'; mukasim vtIIa 'perder', amukasim weiwirapara 'eu perdi minha espingarda'.

\subsubsection{Modos do verbo}

- Indicativo I

- Paradigmas do Indicativo I de verbos intransitivos: 


$\begin{array}{lll}1 & \text { akaru } & \text { 'comi' } \\ 2 & \text { crckaru } & \text { 'comeste' } \\ 13 & \text { urukaru } & \text { 'comemos' } \\ 12(3) & \text { sakaru } & \text { 'comemos' } \\ 23 & \text { pekaru } & \text { 'comestes' } \\ 3 & \text { ukaru } & \text { 'comeu' }\end{array}$

- O tema $k \varepsilon$ vi 'entrar':

$\begin{array}{lll}1 & a k \varepsilon & \text { 'entrei' } \\ 2 & \varepsilon r c k \varepsilon & \text { 'entraste' } \\ 13 & u r u k \varepsilon & \text { 'entramos' } \\ 12(3) & \text { sacke } & \text { 'entramos' } \\ 23 & p \varepsilon k \varepsilon & \text { 'entrastes' } \\ 3 & u k \varepsilon & \text { 'entrou' }\end{array}$

- O tema ur vi 'vir'

$\begin{array}{lll}1 & \text { asor } & \text { 'vim' } \\ 2 & \text { cresor } & \text { 'vieste' } \\ 13 & \text { ur } & \text { 'viemos' } \\ 12(3) & \text { sasor } & \text { 'viemos' } \\ 23 & \text { pesor } & \text { 'viestes' } \\ 3 & \text { uasor } & \text { 'veio' }\end{array}$

- Os temas pinu vi 'peidar' e puti vi 'cagar'.

$\begin{array}{lll}1 & \text { аріпи } & \text { 'peidei' } \\ 2 & \text { сrеріпи } & \text { 'peidaste' } \\ 13 & \text { игиріпи } & \text { 'peidamos' } \\ 12(3) & \text { sаріпи } & \text { 'peidamos' } \\ 23 & \text { реріпи } & \text { 'peidastes' } \\ 3 & \text { иріпи } & \text { 'peidou' }\end{array}$

- Paradigmas do Indicativo I de verbos transitivos:

$1 \quad$ akutuk... 'eu furo...'

$2 \quad$ erckutuk... 'tu furas...'

13 urukutuk... 'nós furamos...'

12(3) sakutuk... 'nós furamos...'

23 pekutuk... 'vós furais...'

$3 \quad$ ukutuk... 'ele furou...' 
- Imperativo

- Paradigmas de verbos intransitivos:

$\mathrm{Na}$ língua Suruí, o Imperativo é construído com o alomorfe $\varepsilon$ - do prefixo $\varepsilon r \varepsilon-$ : son vi Ia 'correr', essn 'corra!', peson 'corram!'; karu vi Ia 'comer', $\varepsilon k a r u$ 'coma!, p pkaru 'comam!'; $k \varepsilon$ vi IIa 'entrar', $\varepsilon k \varepsilon$ 'entre!, $p \varepsilon k \varepsilon$ 'entrem!'.

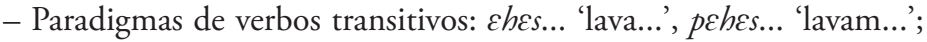

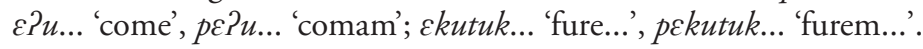

- Gerúndio

- Paradigmas do Gerúndio de verbos intransitivos:

$\begin{array}{lll}1 & \text { wehow } & \text { 'eu indo' } \\ 2 & \text { chow } & \text { 'tu indo' } \\ 13 & \text { uruhow } & \text { 'nós indo' } \\ 12(3) & \text { scnchow } & \text { 'nós indo' } \\ 23 & \text { peschow } & \text { 'vós indo' } \\ 3 & \text { show } & \text { 'ele indo' }\end{array}$

$\mathrm{Na}$ língua Suruí há verbos que, no Gerúndio, ocorrem com um alomorfe de we-, a forma wet-: wetuna, wetsga. (A mesma orientação é válida também para os exemplos intransitivos todos no gerúndio).

- Paradigmas do Gerúndio de verbos intransitivos:

$\begin{array}{lll}1 & \text { wetuna } & \text { 'eu sentando' / 'para eu sentar' } \\ 2 & \text { ctuna } & \text { 'tu sentando' / 'para tu sentares' } \\ 13 & \text { urutuna } & \text { 'nós sentando' / 'para nós sentarmos' } \\ 12(3) & \text { senctuna } & \text { 'nós sentando' / 'para nós sentarmos' } \\ 23 & \text { pesctuna } & \text { 'vós sentando' / 'para vós sentardes' } \\ 3 & \text { stuna } & \text { 'ele sentando' / 'para eles sentarem' }\end{array}$

- Indicativo II

Sobre o modo Indicativo II, Silva (2013, p. 423), citando trabalho de Rodrigues (1958), afirma que: "o modo Indicativo II era uma variedade de indicativo encontrada no Tupinambá, que ocorria quando uma circunstância antecedia um predicado com sujeito de primeira ou de terceira pessoa". Além disso, Cabral e Rodrigues (2003, p. 18) enfatizam que "os verbos em frases independentes iniciadas por uma expressão adverbial, que as condiciona (inclusive os numerais, que nesta língua têm força adverbial) engatilha o 
modo Indicativo II”. Na língua Suruí eles podem ser demonstrados pelos seguintes paradigmas:

- Paradigmas de Indicativo II de verbos intransitivos: i-puraha-s 'dançar', i-ho-s 'ir', i-ata-s 'andar' (cf. subseçâo 6.1.1.2.1).

- Subjuntivo

- Paradigmas de Subjuntivo de verbos intransitivos: $i$-ho-ramu subj 'ir', i-purahas-amu subj 'dançar'. 'matar...',

- Paradigmas de Subjuntivo de verbos transitivos: i-suka-ramu subj

\subsubsection{Negaçâo dos predicados com núcleo verbal e com núcleo nominal descritivo}

Na língua Suruí, a neg o, é responsável pelo imperativo negativo da língua Suruí.

suka vtIa 'matar', csuka 'mata ele!', csuka puhi 'não mata ele!'

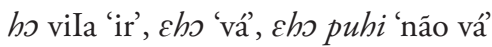

Emuku’om 'levante-o', Emuku’om pubi 'não o levante'

No Gerúndio, no Indicativo II e no Subjuntivo, a negação se exprime mediante o acréscimo do sufixo -eitim 'proibitivo', formador de tema negativo, ao qual se acrescentam os sufixos modais respectivos:

tahi 'gente (está) doente', $t$-ahi-ع?im 'sem dor de gente (de muitos)'

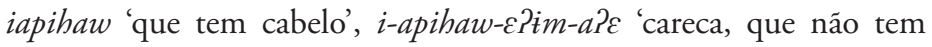
cabelo’

\section{Consideraçóes finais}

Este artigo, ao apresentar um esboço da morfologia da língua Suruí com base na "Estrutura do tupinambá", de Rodrigues (2010 [1981]), por um lado, reúne informaçóes fundamentais sobre a estrutura interna das palavras do Suruí-Aikewára, dos processos que as formam e das categorias gramaticais que as distinguem; e, por outro lado, presta uma homenagem ao grande linguista e professor Aryon Dall'Igna Rodrigues, rememorando, com dados de outra língua Tupí-Guaraní, o seu clássico estudo que orientou 
inúmeros outros estudos descritivos de línguas de diferentes famílias do tronco Tupí, o que tem sido igualmente importante para os trabalhos de reconstrução desse tronco linguístico.

\section{Referências}

CABRAL, Ana Suelly Arruda Câmara. Flexão relacional na família TupíGuaraní. Boletim da Abralin, Fortaleza, n. 25, p. 233-262, dez. 2000.

JENSEN, C. J. S. O desenvolvimento histórico da língua Wayampi. 2004. 183f. Dissertação (Mestrado em Linguística), sob a orientação de Aryon Dall'Igna Rodrigues, Instituto de Estudos da Linguagem, Universidade Estadual de Campinas, Campinas, SP, 1984.

LOPES, J. D. Uma interface da documentação linguística e modelos lexicográficos para línguas indígenas brasileiras: uma proposta para o Suruí-Aikewára. Tese (Doutorado em Linguística), Instituto de Letras, Universidade de Brasília, Brasília, DF, 2014. 599p.

NEVES, Débora David das. A língua Suruí do Tocantins: uma introdução à morfossintaxe. 1999. 50f. Dissertação (Mestrado em Linguística)orientadora Carmen Lúcia R. Rodrigues - Programa de Pós-Graduação em Letras, Centro de Letras e Artes, Universidade Federal do Pará, Belém, 2000.

RODRIGUES, Aryon Dall'Igna. Relaçôes internas na família lingüística Tupí-Guaraní. Revista de Antropologia, v. 27/28, p. 33-53, 1984/1985.

Línguas brasileiras: para o conhecimento das línguas indígenas. São Paulo: Loyola, 1986.

Línguas indígenas: 500 anos de descobertas e perdas. DELTA.

Revista de Documentaçáo em Lingüística Teórica e Aplicada, v. 9, n. 1, p. 83-103, 1993. São Paulo: Associação Brasileira de Lingüística.

A estrutura do Tupinambá. In: CABRAL, Ana Suelly A.C.; RODRIGUES, Aryon D. (Org.). Línguas e Culturas Tupí 2. Campinas, SP: Curt Nimuendajú; Brasília, DF: LALI/UnB, 2010. p. 167-203.

Esboço da morfologia da língua Suruí-Aikewára, com base no clássico trabalho de Rodrigues "A Estrutura do Tupinambá" Jorge Domingues Lopes Recebido em 31/07/2015 Aprovado em 20/10/2015 